
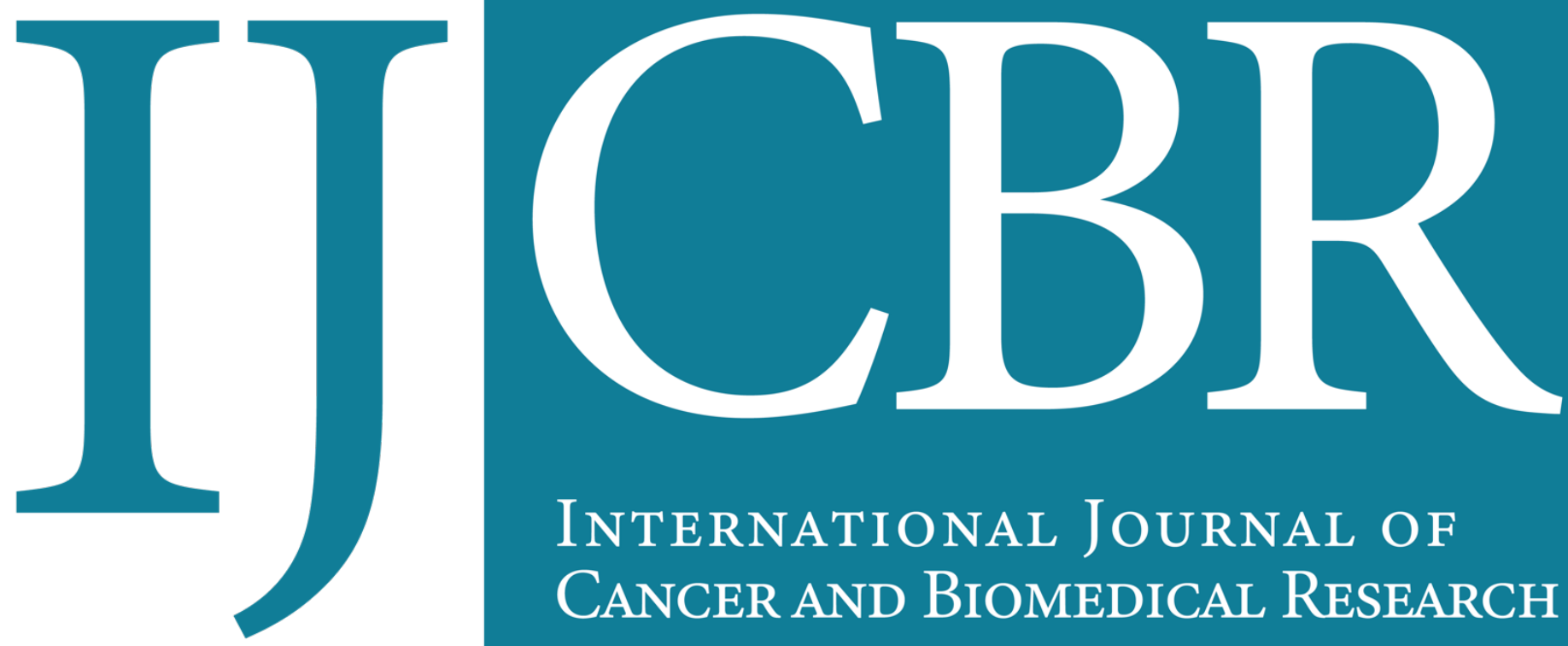

INTERNATIONAL JOURNAL OF CANCER AND BIOMEDICAL RESEARCH

https://jcbr.journals.ekb.eg

Editor-in-chief

Prof. Mohamed Labib Salem, PhD

Prognostic Significance of Trophoblastic Cell Surface Protein 2 (Trop2) Expression in Breast Cancer and Relation to Microvascular Density

Alaa I. Amer, Sara A. Darwish and Fatma MKh Ibrahim 


\title{
Prognostic Significance of Trophoblastic Cell Surface Protein 2 (Trop2) Expression in Breast Cancer and Relation to Microvascular Density
}

\author{
Alaa I. Amer ${ }^{1}$, Sara A. Darwish ${ }^{2}$ and Fatma MKh Ibrahim ${ }^{1}$ \\ ${ }^{1}$ Pathology Department, Faculty of Medicine, Tanta University, Egypt \\ ${ }^{2}$ Clinical Oncology and Nuclear Medicine Department, Faculty of Medicine, Tanta University, Egypt
}

\section{ABSTRACT}

Background: The expression of trophoblastic cell surface protein 2 (Trop2) has been correlated with aggressive behavior in multiple malignant neoplasms. Moreover, it is a promising therapeutic target for several malignancies. However, data on the prognostic significance of Trop2 in breast cancer is insufficient. The current work aimed to study the prognostic significance of Trop2 expression in breast cancer and its correlation with microvascular density (MVD). Patients and Methods: Immunohistochemical expression of Trop2 was assessed in 70 cases of breast cancer and its relation to clinicopathological parameters, MVD (as assessed by CD34 immunostaining) and patient's survival was evaluated. Results: High Trop2 expression was detected in 44 cases $(62.9 \%)$ of the studied cases. The expression of Trop2 was significantly related to tumor size, lymph node stage, lymphovascular invasion, tumor grade and high Ki67 index $(\mathrm{P}<0.05)$. Moreover, the expression of Trop2 was significantly higher in triple-negative breast carcinoma (TNBC) than other molecular subtypes $(\mathrm{P}=0.015)$. Additionally, Trop2 expression was significantly correlated to MVD $(r=0.845)$. Both overall survival and disease-free survival were significantly shorter in the high Trop2 group than low Trop2 group $(\mathrm{P}=0.008,0.021)$, respectively. Conclusion: Trop2 can be considered as a poor prognostic marker and potential therapeutic target for breast cancer, mainly the triple-negative subtype. Since Trop2 expression was significantly correlated to MVD, we speculate that enhancement of angiogenesis could be one of the mechanisms involved in Trop2 induced tumor invasion and metastasis.

Keywords: breast cancer, Triple-negative breast cancer, Trop2, microvascular density

Editor-in-Chief: Prof. M.L. Salem, PhD - Article DOI: 10.21608/jcbr.2021.68609.1189

\section{ARTICLE INFO}

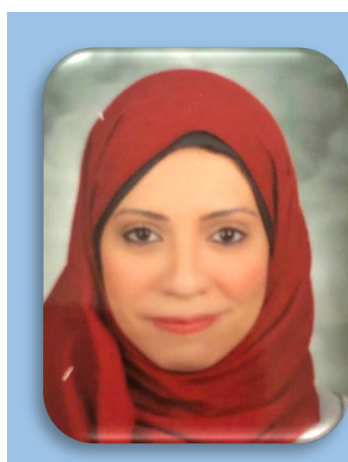

Article history

Received: March 19, 2021

Revised: May 16, 2021

Accepted: May 25, 2021

Correspondence to:

Alaa Ibrahim Amer, MD

Pathology Department,

Faculty of Medicine,

Tanta University, Egypt

Tel: 01003642577

E-Mail: alaa.amer@med.tanta.edu.eg

\section{INTRODUCTION}

Breast cancer (BC) is the most common malignancy among females, comprising around $25 \%$ of all female cancers, and the leading cause of cancer-related mortality (Sung et al., 2021). Breast cancer is a heterogenous disease with several molecular subtypes. Triple-negative breast carcinoma (TNBC) is an aggressive subtype of breast cancer, which accounts for $10-15 \%$ of all BC cases with more prevalence among young African American females (Cocco et al., 2020).

The further molecular analysis emphasizes the heterogenicity of TNBC, which can explain variable prognosis and response to therapy among patients. Patients with TNBC are not candidates for hormonal therapy or Her2/neu directed therapy, therefore their treatment depends mainly on chemotherapy (Zhao et al., 2018). The available prognostic markers for $B C$ are not sufficient to predict patient outcome and response to therapy (Abd El-Azeem, 2021). Hence, research about new prognostic biomarkers and novel therapeutic targets for $B C$, mainly the aggressive TNBC subtype, is mandatory (Ambrogi et al., 2014).

Trophoblastic cell surface protein 2 (Trop2), also known as tumor associated calcium signal transductor (TACSTD2) or gastrointestinal 
antigen (GA73), is a cell surface glycoprotein which was first identified on invasive human trophoblastic cells (Ning b et al., 2013). Trop2 shares structural similarity with epithelial cell adhesion molecule (EPCAM), another member of TACSTD gene family, which may explain its function as a cell adhesion molecule (Lin et al, 2013). Previous studies illustrated the association between Trop2 expression and the aggressive behavior of several malignant neoplasms (Shvartsur and Bonavida, 2015). However, data on the prognostic significance of Trop2 in breast cancer is limited. Trop2 can mediate intercellular adhesion, tumor proliferation and invasion through interaction with several intracellular ligands. Moreover, it is involved in epithelial-mesenchymal transition (EMT), an important process for tumor invasion and metastasis (Zhao et al., 2018). Trop2 is considered an attractive therapeutic target due to its frequent expression in a range of malignant tumors, with less common expression in normal human tissues (Lin et al., 2013).

Pathological angiogenesis is critical for malignant transformation and metastasis. Malignant cells need newly formed vessels for their nutrition and invasion. Angiogenic switch in malignant tumors is a complex process, regulated by several proangiogenic and antiangiogenic factors (Zhou et al., 2010). The exact mechanism and promoting factors of angiogenesis in breast cancer remain to be illustrated. Microvascular density (MVD) is the most frequently used quantitative measurement of pathological angiogenesis. High MVD has been correlated with poor prognosis in several malignancies including breast cancer (Mackey et al., 2012 and Kraby et al., 2015). Anti-angiogenic drugs have been approved to decrease tumor growth and progression in several malignancies including metastatic breast cancer (Chen et al., 2015)

The current work aimed to study the expression of Trop2 in breast cancer to assess its prognostic significance and relation to patient survival. Additionally, we aimed to study the relation between Trop2 expression in breast cancer and MVD.

\section{PATIENTS AND METHODS}

\section{Study design}

The current prospective study included 70 cases of breast carcinoma. The study was carried out in pathology and clinical oncology departments, Faculty of Medicine, Tanta University during the period from January 2018 to February 2021. The study protocol was approved by the Faculty Research Ethics Committee (REC) before the conduction of the study (approval code number: 34509).

The inclusion criteria for this study were histopathological proof of nonmetastatic breast cancer, age $\geq 18$ years, World Health Organization (WHO) performance status of 0 or 1 , adequate organs function and the presence of proper histological specimens with sufficient tumor tissue. Cases fulfilling the inclusion criteria were enrolled in the study at the beginning of work. Patients with serious illness (like significant heart disease or myocardial infarction) within 3 months, history of other malignancy, pregnant and lactating patients were excluded from the study.

\section{Patient evaluation, treatment and follow-up}

Careful history taking and complete clinical examination were done. Patients were evaluated to exclude metastasis through laboratory and radiological investigations including, plain $\mathrm{x}$-ray chest, breast mammography, ultrasound examination of abdomen and pelvis, bone scan, and if needed CT scan of the chest.

Patients were subjected to surgical interference in the form of modified radical mastectomy or conservative breast surgery and the surgical specimens were referred to the Pathology department for evaluation. The surgical decision was determined according to the current practice guidelines in the treatment of breast cancer depending on the clinical stage, tumor size and lymph node status. Patients with single, small-sized tumor masses and early tumor stage (Stage I or II) were operated by conservative breast surgery. While, modified radical mastectomy was performed in cases with more advanced stage, larger tumor size, multiple masses and centrally positioned tumors. 
Patients received adjuvant or neoadjuvant treatment according to clinical stage and current guidelines (chemotherapy \pm radiotherapy \pm hormonal treatment with or without target therapy (according to Her2/neu expression). Main chemotherapy regimens included anthracyclines, taxanes, cyclophosphamide and 5-fluorouracil. Trastuzumab was administered only in patients with Her2/neu expressing tumors. Patients were evaluated every 3 months during the first year, every 6 months during the second year and once a year after. Patient's data as regard age, tumor size, lymph node status, hormonal receptor (ER/PR), HER2neu expression, histological grade, type of treatment, diseasefree survival (DFS) and overall survival (OS) were recorded.

Overall survival (OS) was defined as the interval between the date of start of treatment and date of last visit or date of death, independent of the cause of death. Disease-free survival (DFS) was defined as the interval between the date of start of treatment and either recurrence of breast cancer or occurrence of metastasis.

\section{Histopathological evaluation}

The histopathological subtype of the studied cases was determined according to the WHO criteria (Lakhani et al., 2012). Tumors were graded according to The Nottingham grading system (Pradhan et al., 2017) and the pathological stage was determined according to TNM system of the American Joint Committee of Cancer staging (AJCC) (Hortobagy et al., 2018). Cases were classified according to modern molecular classification into luminal $A$, luminal B, Her2 neu enriched and triplenegative subtypes (Goldhirsch et al., 2011).

\section{Immunohistochemical staining}

Paraffin blocks sections of $5 \mu \mathrm{m}$ thickness, on positively charged slides, were left to dry for 30 minutes at $37^{\circ} \mathrm{C}$. Deparaffinization and antigen retrieval were performed in Dako PT Link unit using high and low pH EnVisionTM FLEX antigen retrieval solutions reaching a temperature of $97^{\circ} \mathrm{C}$ for $20 \mathrm{~min}$ before cooling. Immunohistochemical staining was performed on the Dako Autostainer Link 48. The internal peroxidase activity was blocked, followed by incubation with primary antibodies for $30 \mathrm{~min}$ with subsequent addition of horseradish peroxidase (HRP) polymer reagent for $20 \mathrm{~min}$. Diaminobenzidine (DAB) was applied as chromogen. Finally, the slides were counterstained with hematoxylin. The following primary antibodies were used in this study: Trop2; a rabbit monoclonal antibody (Cat. No. 2TU022-13, ready to use, Quartett, Berlin, Germany), CD34; a mouse monoclonal antibody (Cat. No. M7165; 1: 100 dilution Dako, USA).

\section{Interpretation of immunohistochemical staining Evaluation of Trop2 immunoreactivity}

Trop2 immunoreactivity was assessed as positive membranous staining in the neoplastic cells, and the staining was semiquantitatively scored by multiplying the percentage of positively stained cells and the intensity of staining. The percentage of positive tumor cells was classified as following, negative (0), $<10 \%$ (1), $10-50 \%$ (2), $51-80 \%$ (3), or $>80 \%$ (4). The staining intensity was scored as follows, no staining (0), weak (1), moderate (2), or strong (3). The final score ranged from 0-12 and was then classified into, low expression (0-4) and high expression (6-12) (Ninga et al., 2013).

\section{Evaluation of MVD}

CD34 immunostaining was used to evaluate tumor microvascular density (MVD).CD34 stained tissue sections were examined at low power microscopy to identify intratumoral hyper vascularized areas (hot spots). The microvessels were then counted in three high power fields (HPF) $(x 400)$ of hot spots using the plug-in 'cell counter' added to the Image J software (inspired by National Institute of Health, USA]. CD34 positive isolated endothelial cells or groups of cells with or without lumen were considered as countable microvessels, and the mean vessel count in 3 HPF was considered as the mean MVD. For the statistical purpose in this study, cases with the mean vascular count of $\leq 15 / \mathrm{HPF}$ were considered as low MVD, while cases with the mean vascular count of $>15 / \mathrm{HPF}$ were considered as high MVD (Chen et al., 2015).

\section{Statistical analysis}

Statistical analysis was performed using Statistical Package for Social Science (SPSS 
version 23). Data were expressed in terms of frequencies and percentages for categorical variables. For numerical data, mean and standard deviation (+SD) or median and range were calculated. For comparing categorical data, Chi-square (X2) test was performed. Monto Carlo and Fisher Exact tests were used when appropriate. Student t-test was used to compare two groups for normally distributed quantitative variables while Mann Whitney test was used to compare between two groups for not normally distributed quantitative variables. Kaplan-Meier survival curves were generated and compared using the log-rank test. Correlation between Trop2 expression score and MVD was evaluated using Spearman correlation coefficient. The significance of various prognostic factors was evaluated in a multivariate Cox proportional hazards regression model. $\mathrm{P}$ values of $<0.05$ were considered statistically significant.

\section{RESULTS}

\section{Clinicopathological characteristics}

The present study included 70 patients diagnosed with nonmetastatic breast carcinoma. The mean age of the studied cases was $57.6 \pm 9.9$ years (range 33-74 years). In 20 patients (28.6\%), the tumor size was $\geq 5 \mathrm{~cm}$ in its largest diameter (T3 and T4). Grade II carcinoma constituted 50 cases $(71.4 \%)$, while triplenegative breast cancer cases constituted 20 cases (28.6\%) of the studied cases. Lymphovascular invasion was detected in 26 cases $(37.1 \%)$, while lymph node metastasis was detected in 44 (62.9\%) of the studied cases. All patients underwent surgical interference either in form of modified radical mastectomy (MRM) in 26 patients (37.1\%) or conservative breast surgery in 44 patients (62.9\%). Patients received adjuvant or neoadjuvant chemotherapy according to guidelines and clinical stage. The main chemotherapy regimens included anthracyclines in 10 patients (14.3\%), taxanes in 15 patients $(21.4 \%)$, the combination of anthracyclines and taxanes in 45 patients (64.3\%). Additionally, 67 patients (95.7\%) received radiotherapy. Trastuzumab was administered in 14 patients (20\%) with HER2/neu+ tumors. Patients with positive hormonal receptors (ER, $P R$ ) received endocrine therapy with letrozole, aramidex, or tamoxifen.
The clinicopathological features of the studied breast cancer cases are illustrated in Table 1.

Table 1. Clinicopathological features of studied cases

\begin{tabular}{|c|c|}
\hline & No (\%) \\
\hline Age (Mean \pm SD.) & $57.6 \pm 9.9$ \\
\hline$<50$ years & $15(21.4 \%)$ \\
\hline$\geq 50$ years & $55(78.6 \%)$ \\
\hline \multicolumn{2}{|l|}{ Performance status (PS) } \\
\hline 0 & $49(70 \%)$ \\
\hline 1 & $21(30 \%)$ \\
\hline \multicolumn{2}{|l|}{ Menopause } \\
\hline Pre & $21(30 \%)$ \\
\hline Post & $49(70 \%)$ \\
\hline \multicolumn{2}{|l|}{ Tumor size (T) } \\
\hline $\mathrm{T} 1$ & $27(38.6 \%)$ \\
\hline $\mathrm{T} 2$ & $23(32.9 \%)$ \\
\hline T3 & $13(18.6 \%)$ \\
\hline T4 & $7(10 \%)$ \\
\hline \multicolumn{2}{|l|}{ Lymph node status (N) } \\
\hline NO & $26(37.1 \%)$ \\
\hline N1 & $20(28.6 \%)$ \\
\hline N2 & $16(22.9 \%)$ \\
\hline N3 & $8(11.4 \%)$ \\
\hline \multicolumn{2}{|l|}{ Grade } \\
\hline II & $50(71.4 \%)$ \\
\hline III & $20(28.6 \%)$ \\
\hline ER positive & $47(67.1 \%)$ \\
\hline PR positive & $43(61.4 \%)$ \\
\hline Her2 positive & $14(20 \%)$ \\
\hline \multicolumn{2}{|l|}{ TNBC } \\
\hline Yes & $20(28.6 \%)$ \\
\hline No & 50 (71.4\%) \\
\hline $\begin{array}{l}\text { Lympho Vascular invasion } \\
\text { Present }\end{array}$ & $26(37.1 \%)$ \\
\hline Absent & $44(62.9 \%)$ \\
\hline \multicolumn{2}{|l|}{ Ki67 } \\
\hline$<14$ & $24(34.3 \%)$ \\
\hline$>14$ & $46(65.7 \%)$ \\
\hline \multicolumn{2}{|l|}{ Trop2 expression } \\
\hline High & $44(62.9 \%)$ \\
\hline low & $26(37.1 \%)$ \\
\hline \multicolumn{2}{|l|}{ MVD } \\
\hline Low & $31(44.3 \%)$ \\
\hline High & $39(55.7 \%)$ \\
\hline \multicolumn{2}{|l|}{ surgery } \\
\hline MRM & $26(37.1 \%)$ \\
\hline Conservative & $44(62.9 \%)$ \\
\hline Radiotherapy & 67 (95.7\%) \\
\hline Hormonal & $54(77.1 \%)$ \\
\hline \multicolumn{2}{|l|}{ Chemotherapy } \\
\hline Anthracyclin & $10(14.3 \%)$ \\
\hline Taxins & $15(21.4 \%)$ \\
\hline Anthracyclin+ Taxins & 45 (64.3\%) \\
\hline \multicolumn{2}{|l|}{ DFS } \\
\hline No relapse & $51(72.9 \%)$ \\
\hline Relapse & 19 (27.1\%) \\
\hline \multicolumn{2}{|l|}{ OS } \\
\hline Alive & $56(80 \%)$ \\
\hline Dead & $14(20 \%)$ \\
\hline
\end{tabular}




\section{Relation between immunohistochemical expression of Trop2 and clinicopathological parameters}

Trop2 expression was detected as brown membranous staining in the neoplastic cells (Figure 1). Out of the studied 70 cases, high Trop2 expression was detected in 44 cases (62.9\%) (Table 1). Trop2 expression was significantly related to tumor size $(P=0.001)$, lymph node stage $(P=0.001)$ and high Ki67 proliferation index ( $P$ value $<0.001)$. In addition, there was a significant relation between Trop2 expression and the presence of lymphovascular invasion $(P=0.004)$ as $84.6 \%$ of the cases with lymphovascular invasion showed high Trop2 expression. Trop2 expression was also significantly related to histological tumor grade $(P=0.003)$. Among grade III cases, $90 \%$ of cases exhibited high Trop2 expression, while 52\% of grade II tumors showed high Trop2 expression. The expression of Trop2 was significantly higher in TNBC cases ( $P=0.015)$, as $17 / 20$ (85\%) of TNBC cases showed high Trop2 expression. No significant relation was detected between Trop2 expression and patients age $(P=0.064)$, performance state $(\mathrm{P}=0.084)$ or menopause status $(\mathrm{P}=0.331)$ as shown in Table 2 .

\section{Correlation between Trop2 expression and MVD in breast cancer cases}

High Trop2 expression was detected in $87.2 \%$ of cases with high MVD, while $32.3 \%$ of cases with low MVD showed high Trop2 expression. The expression of Trop2 expression was significantly correlated to MVD as measured by CD34 expression $(r=0.845, p<0.001)$, as presented in Figures 2 and 3.

\section{Prognostic value of Trop2 expression in breast carcinoma}

The median follow-up period was 36 months (ranged from 18 to 38 months). Relapse occurred in 19 patients (27.1\%). Out of the 44 cases with high Trop2 expression, 16 patients (36.4\%) developed relapse, while only 3 patients (11.5\%) of the low Trop2 group developed relapse. A statistically significant distribution of relapses in favor of the high Trop2 group was detected $(P=0.024)$.
Mortality occurred in 14 patients (20\%), thirteen patients (92.9\%) were in the high Trop 2 group and only one patient mortality (7.1\%) was reported in the low Trop2 group with a statistically significant distribution of mortality in favor of high Trop2 group $(P=0.009)$. Both OS and DFS rates were significantly lower in high Trop2 patients in comparison to low Trop2 patients $(P$ value $=0.008$ and 0.021, respectively), as shown in Figure 4.

On performing univariate analysis of the prognostic markers in relation to OS, high Trop2, tumor size, high nodal stage, high tumor grade, high Ki 67 and TNBC were found to be statistically significant, while none of them was found to be statistically significant in multivariate analysis, as shown in Table 3.

As regard, the analysis of the prognostic parameters in relation to disease-free survival, tumor size and histological grade were statistically significant on multivariate and univariate analysis. Meanwhile, high Trop2 expression, high tumor grade, TNBC, lymphovascular invasion and increased MVD were found to be statistically significant in univariate but not in multivariate analysis, as illustrated in Table 4.

\section{DISCUSSION}

Metastasis and invasion are the main causes of cancer related morbidity and mortality. Hence, research about biomarker involved in tumor spread and invasion is essential to improve patient outcome. Such markers can serve as potential therapeutic targets as well (Cocco et al., 2020). Trop2 is a cell surface glycoprotein, which is potentially involved in development of metastatic phenotype in several malignant neoplasm (Alberti et al., 2020). In the present work, we studied the expression of Trop2 in 70 cases of breast cancer to assess its prognostic significance and its relation to clinicopathological parameters, mainly MVD.

In the present study, membranous expression of Trop2 was detected in $62.9 \%$ of the studied cases, while focal cytoplasmic expression was detected in some cases. Although Trop2 is mainly a cell surface protein, it is structurally composed of extracellular and intracellular domains. 
Table 2. Relation between Trop2 and clinicopathological characters of breast carcinoma cases.

\begin{tabular}{|c|c|c|c|c|}
\hline & \multicolumn{2}{|c|}{ Trop2 expression } & & \multirow{2}{*}{ p } \\
\hline & Low $(n=26)$ & High $(n=44)$ & & \\
\hline \multicolumn{3}{|l|}{ Age } & \multirow{3}{*}{$\mathrm{t}=1.886$} & \multirow{3}{*}{0.064} \\
\hline$<50$ years & $4(26.7 \%)$ & $11(73.3 \%)$ & & \\
\hline$\geq 50$ years & $22(40 \%)$ & $33(60 \%)$ & & \\
\hline \multicolumn{3}{|c|}{ Performance status } & \multirow{3}{*}{$x^{2}=2.984$} & \multirow{3}{*}{0.084} \\
\hline 0 & $15(30.6 \%)$ & $34(69.4 \%)$ & & \\
\hline 1 & $11(52.4 \%)$ & $10(47.6 \%)$ & & \\
\hline \multicolumn{3}{|c|}{ Menopause } & \multirow{3}{*}{$x^{2}=0.944$} & \multirow{3}{*}{0.331} \\
\hline Pre & $6(28.6 \%)$ & $15(71.4 \%)$ & & \\
\hline Post & $20(40.8 \%)$ & $29(59.2 \%)$ & & \\
\hline \multicolumn{3}{|c|}{ Tumor size (T) } & \multirow{5}{*}{$x^{2}=15.991^{*}$} & \multirow{5}{*}{$0.001^{*}$} \\
\hline $\mathrm{T} 1$ & $16(59.3 \%)$ & $11(40.7 \%)$ & & \\
\hline $\mathrm{T} 2$ & $9(39.1 \%)$ & $14(60.9 \%)$ & & \\
\hline T3 & $0(0 \%)$ & $13(100 \%)$ & & \\
\hline T4 & $1(14.3 \%)$ & $6(85.7 \%)$ & & \\
\hline \multicolumn{3}{|c|}{ Lymph node status (N) } & \multirow{5}{*}{$x^{2}=15.834^{*}$} & \multirow{5}{*}{$0.001^{*}$} \\
\hline NO & $11(42.3 \%)$ & 15 (57.7\%) & & \\
\hline N1 & $13(65 \%)$ & $7(35 \%)$ & & \\
\hline N2 & $2(12.5 \%)$ & $14(87.5 \%)$ & & \\
\hline N3 & $0(0 \%)$ & $8(100 \%)$ & & \\
\hline \multicolumn{3}{|l|}{ Grade } & \multirow{3}{*}{$x^{2}=8.636^{*}$} & \multirow{3}{*}{$0.003^{*}$} \\
\hline II & 24 (48.0\%) & $26(52.0 \%)$ & & \\
\hline III & $2(10.0 \%)$ & $18(90.0 \%)$ & & \\
\hline \multicolumn{5}{|l|}{ TNBC } \\
\hline Yes & $3(15 \%)$ & $17(85 \%)$ & $x^{2}=5.880^{*}$ & \\
\hline No & $23(46 \%)$ & $27(54 \%)$ & \multirow[t]{5}{*}{$x^{2}=8.388^{*}$} & $0.015^{*}$ \\
\hline Lymphovas & ular invasion & & & \multirow{3}{*}{$0.004^{*}$} \\
\hline present & $4(15.4 \%)$ & $22(84.6 \%)$ & & \\
\hline Absent & $22(50 \%)$ & $22(50 \%)$ & & \\
\hline \multicolumn{3}{|l|}{ Ki67 } & & \\
\hline$<14$ & 18 (75.0\%) & $6(25.0 \%)$ & \multirow{2}{*}{$X^{2}=22.419 *$} & \multirow{2}{*}{$<0.001^{*}$} \\
\hline$>14$ & $8(17.4 \%)$ & $38(82.6 \%)$ & & \\
\hline MVD & & & & \\
\hline Low & 21 (67.7\%) & $10(32.3 \%)$ & $x-232148^{*}$ & $<0001^{*}$ \\
\hline High & $5(12.8 \%)$ & 34 (87.2\%) & $x^{2}=22.314^{8}$ & $<0.001$ \\
\hline DFS & & & & \\
\hline No relapse & $23(45.1 \%)$ & $28(54.9 \%)$ & $x^{2-5} 092 *$ & \\
\hline Relapse & $3(15.8 \%)$ & $16(84.2 \%)$ & $x^{2}=5.093^{\prime \prime}$ & $0.024^{*}$ \\
\hline OS & & & & \\
\hline Alive & $25(44.6 \%)$ & $31(55.4 \%)$ & & \\
\hline Dead & $1(7.1 \%)$ & $13(92.9 \%)$ & $x^{2}=6.746$ & $0.009^{*}$ \\
\hline
\end{tabular}

$\chi^{2}$ : Chi-square test, MC: Monte Carlo, FE: Fisher Exact test, U: Mann Whitney test, $\mathrm{P}: \mathrm{P}$ value for comparing between the studied groups.

The Intracellular portion is released from the membrane into the cytoplasm to regulate several intracellular pathways, while the extracellular domain is mainly expressed in the cellular membrane, serving in tight junction regulation (Shvartsur and Bonavida, 2015).

Ambrogi et al. (2014) also demonstrated the presence of cytoplasmic and membranous portions of Trop2 in breast cancer tissue specimens they reported that membranous expression of Trop2 was associated with poor prognostic parameters and shorter patient survival, while cytoplasmic expression was associated with better prognosis and longer progression-free survival. They suggested that the membranous portion is the mature active part of Trop2, while the retained intracellular portion is the immature inactive part.

In the present study, a significant relation was detected between Trop2 expression and high 
Table 3. Univariate and multivariate COX regression analysis of prognostic parameters for overall survival in breast cancer cases.

\begin{tabular}{|c|c|c|c|c|}
\hline & \multicolumn{2}{|r|}{ Univariate } & \multicolumn{2}{|r|}{ \#Multivariate } \\
\hline & p & HR (95\% C.I) & p & HR (95\% C.I) \\
\hline Trop2 (Low Vs high) & $0.032^{*}$ & $9.281(1.213-71.0)$ & 0.322 & $3.638(0.282-46.923)$ \\
\hline Age & 0.830 & $0.994(0.944-1.048)$ & & \\
\hline Performance status (PS) & 0.347 & $1.697(0.564-5.102)$ & & \\
\hline Menopause (post) & 0.832 & $1.134(0.355-3.617)$ & & \\
\hline Tumor size (T1,2 Vs T3,4) & $0.002^{*}$ & $5.568(1.862-16.652)$ & 0.688 & $0.628(0.065-6.058)$ \\
\hline Node status ( 0 vs $1+2+3$ ) & $0.041^{*}$ & $8.315(1.087-63.607)$ & 0.277 & $3.458(0.369-32.396)$ \\
\hline Grade (GII Vs GIII) & $0.046^{*}$ & $2.909(1.017-8.320)$ & 0.462 & $2.026(0.309-13.290)$ \\
\hline TNBC & $0.020^{*}$ & $3.527(1.223-10.171)$ & 0.881 & \\
\hline Lympho vascular invasion & 0.056 & $2.818(0.975-8.147)$ & & \\
\hline Ki67 (low Vs. high) & $<0.001^{*}$ & $1.062(1.027-1.097)$ & 0.898 & $1.006(0.915-1.106)$ \\
\hline MVD 9 (Low Vs high) & 0.139 & $2.403(0.752-7.684)$ & & \\
\hline
\end{tabular}

Table 4. Univariate and multivariate COX regression analysis of prognostic parameters for diseases free survival in breast cancer cases.

\begin{tabular}{|l|c|c|c|c|}
\hline & \multicolumn{2}{|c|}{ Univariate } & \multicolumn{1}{c|}{ \#Multivariate } \\
\hline Trop2 (Low Vs high) & $0.033^{*}$ & $3.820(1.111-13.130)$ & 0.608 & $0.635(0.112-3.592)$ \\
\hline Age & 0.822 & $0.995(0.950-1.041)$ & & \\
\hline Performance status (PS) & 0.753 & $1.168(0.443-3.077)$ & & \\
\hline Menopause (post) & 0.567 & $0.761(0.300-1.935)$ & & \\
\hline Tumor size (T1,2 Vs T3,4) & $<0.001^{*}$ & $21.806(6.302-75.457)$ & $0.042^{*}$ & $5.498(1.067-28.328)$ \\
\hline Node status (0 vs 1+2+3) & $0.012^{*}$ & $13.130(1.751-98.439)$ & 0.639 & $1.860(0.139-24.815)$ \\
\hline Grade (GII Vs GIII) & $0.001^{*}$ & $4.564(1.828-11.398)$ & $0.018^{*}$ & $5.643(1.347-23.647)$ \\
\hline TNBC & $0.032^{*}$ & $2.680(1.087-6.605)$ & 0.207 & $2.208(0.645-7.558)$ \\
\hline Lympho vascular invasion & $0.008^{*}$ & $3.555(1.395-9.061)$ & 0.396 & $1.840(0.450-7.517)$ \\
\hline Ki67 (low Vs. high) & 0.056 & $3.328(0.969-11.429)$ & & \\
\hline MVD 9 (Low Vs high) & $0.029^{*}$ & $3.433(1.138-10.355)$ & 0.505 & $0.455(0.045-4.623)$ \\
\hline
\end{tabular}

tumor grade, advanced tumor stage, lymph node metastasis and the presence of lymphovascular invasion. High expression of Trop2 was also significantly related to shorter OS and DFS. Our results matched those of Lin et al. (2013) and Amborgi et al. (2014). Kluger et al. (2004) also demonstrated that Trop2 gene was amongst the upregulated genes in breast cancer cell lines after induction of invasive phenotype.

Moreover, Zhao et al. (2018) reported high expression of Trop2 and low expression of $\mathrm{E}$ cadherin in breast cancer tissue specimens and cell lines. Furthermore, they reported that Trop2 ${ }^{+}$-E-cadherin ${ }^{-}$group of their studied cohort, was associated with the highest metastatic rate, worst prognosis and shortest overall survival. They concluded that Trop2 is a potential promoting factor for tumor invasion through enhancement of epithelial mesenchymal transition (EMT).

The promoting role of Trop2 on tumor metastasis was further supported by Alberti et al., 2020. They studied a large series of variable malignant tumors, including breast, colon, lung, ovarian and gastric cancer, and demonstrated that the activation of the cytoplasmic tail of Trop2 can stimulate release of E-cadherin from cytoskeleton with activation of $B$ catenin and subsequent enhancement of EMT.

In agreement with Lin et al. (2013), the current study revealed a significant relation between Trop2 expression and high KI67 proliferation index. By contrast, in the cohort studied by Zhao et al. (2018) Trop2 expression was not significantly related to proliferation index. Trop2 enhances cellular proliferation and cell cycle stimulation through the regulation of several intracellular pathways including WNT/B catenin signaling cascade. Moreover, Trop2 mRNA can fuse with Cyclin D1 mRNA, a process that increases the stability of cyclin. Cyclin D1 is an important regulatory cell cycle transcription factor. The cyclin D1-Trop2 mRNA complex is considered a potent oncogene with an ability 

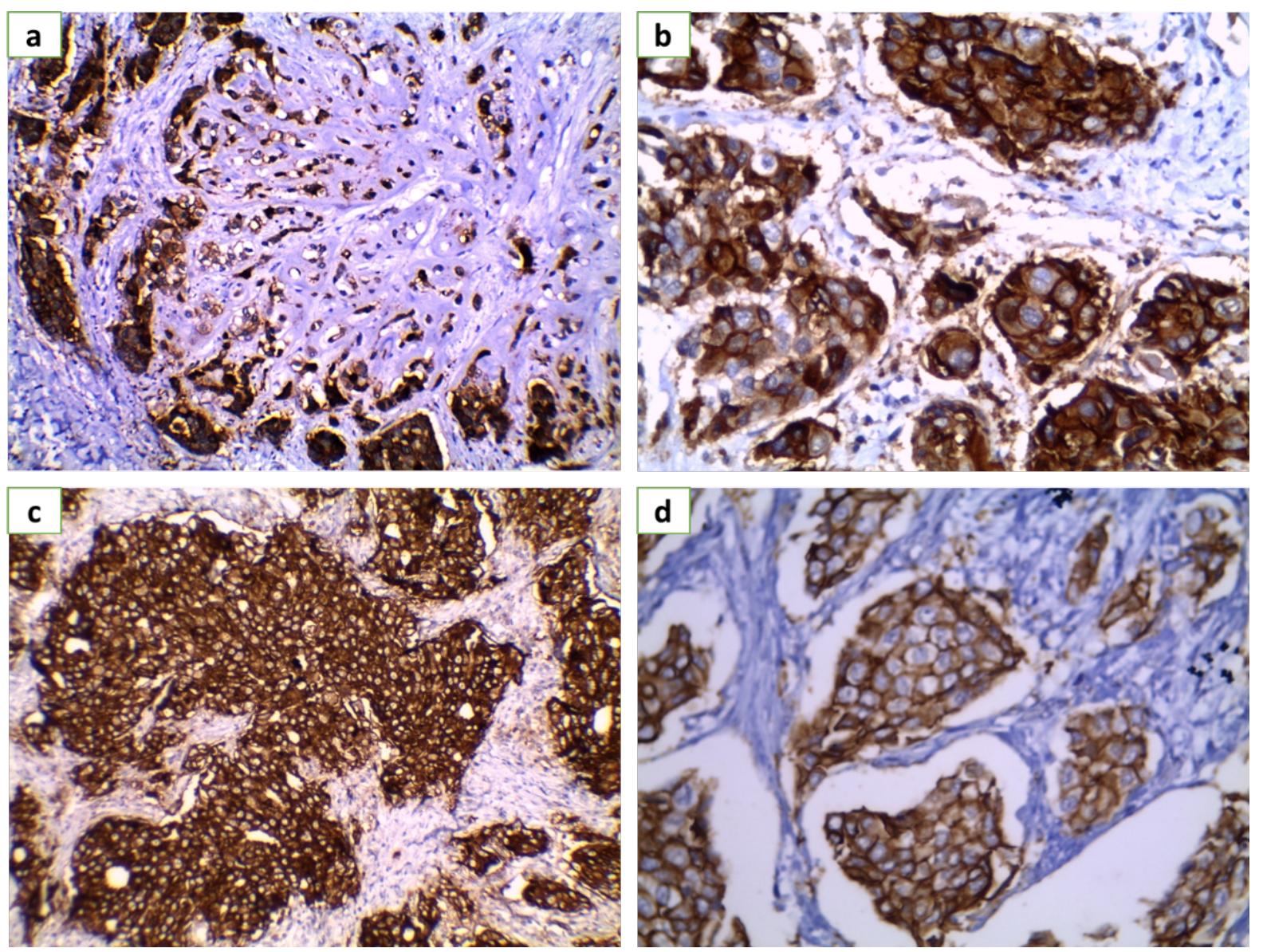

Figure 1. Immunohistochemical expression of Trop2 in breast carcinoma (Brown membranous staining in the neoplastic cells points to Trop2 expression): (a) high expression in metaplastic breast carcinoma with chondroid differentiation (x200), (b) higher magnification of the previous image(x400), (c) high expression in grade III infiltrating ductal carcinoma (IDC) with medullary features(x200), (d) high expression in IDC grade II (x400).

to transform cells in vitro and to induce aggressive behavior in several malignancies (Lin et al., 2013).

In contrast to our results, Huang et al. (2005) reported low levels of Trop2, as measured by quantitative RT-PCR, in breast cancer cell lines. Moreover, they did not detect a significant relation between Trop2 expression and clinicopathological parameters. The discrepancy may be due to different study designs and different methods applied to assess Trop2 expression.

As regards the expression of Trop2 in different molecular subtypes of breast cancer, the current study revealed that Trop2 expression was significantly higher among TNBC cases than other molecular subtypes. This result was consistent with Zhao et al. (2018). On the contrary, in the studies conducted by Huang et al. (2005) and Lin et al. (2013), Trop2 expression was not related to the hormonal receptor or Her2neu expression. Yet, further analysis of the cohort studied by Huang et al. (2005) revealed that Trop2 was more expressed in ER negative/Her2neu positive tumors than other subtypes.

Angiogenesis is one of the hallmarks of cancer. Tumor angiogenesis is enhanced by proangiogenic factors, which can be released from tumor cells and stimulate existing vessels to proliferate and infiltrate tumor tissue. Tumor angiogenesis is an attractive therapeutic target in multiple malignancies including breast cancer (Mackey et al., 2012). Increased MVD in breast cancer has been correlated with a higher rate of metastasis, recurrence and shorter overall survival. Hence, MVD is considered as an independent prognostic factor in multiple molecular subtypes of breast cancer (Kraby et al., 2017). 

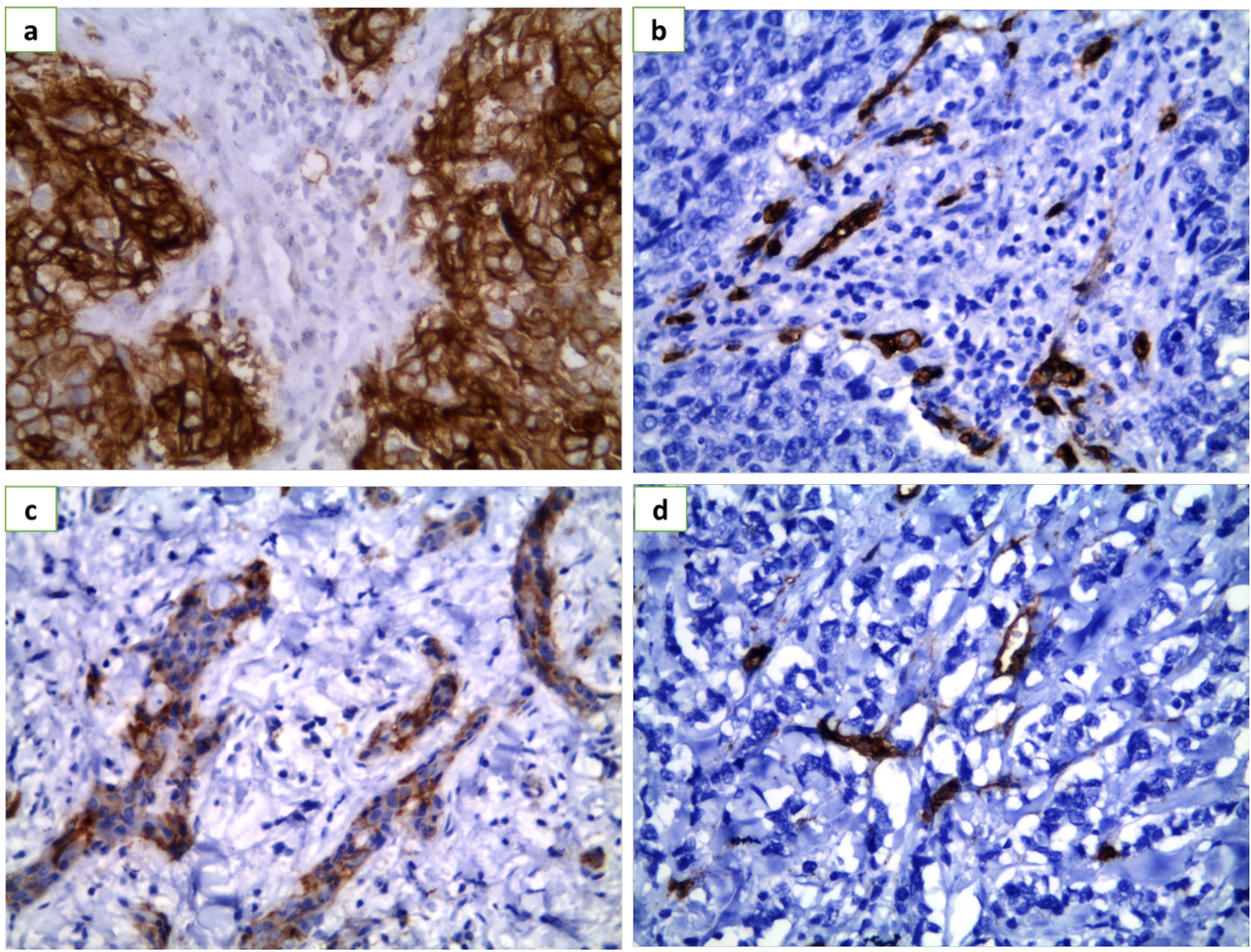

Figure 2. Trop2 expression in relation to MVD in breast carcinoma ( $x 400)$ : a, b) grade III IDC with medullary features exhibiting high Trop2 expression and high MVD, a) strong membranous Trop2 expression in IDC cells b) CD34 immunostaining highlights numerous intratumoral microvessels (high MVD),c, d) grade II IDC exhibiting low Trop2 expression and low MVD, c) low Trop2 expression in IDC cells d) CD34 immunostaining highlights few intratumoral microvessels (low MVD).

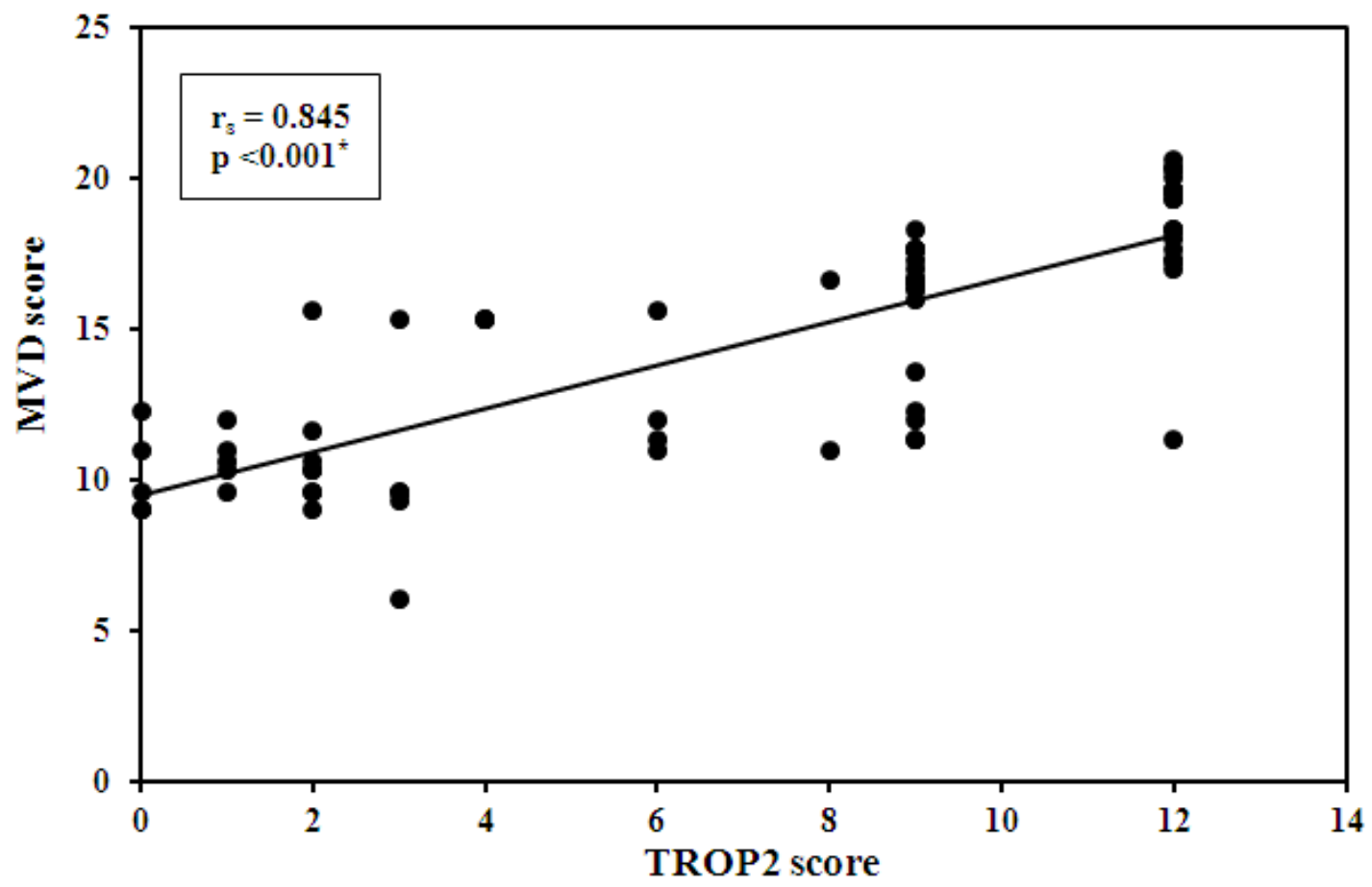

Figure 3. Correlation between Trop2 expression score and MVD in breast carcinoma cases. 


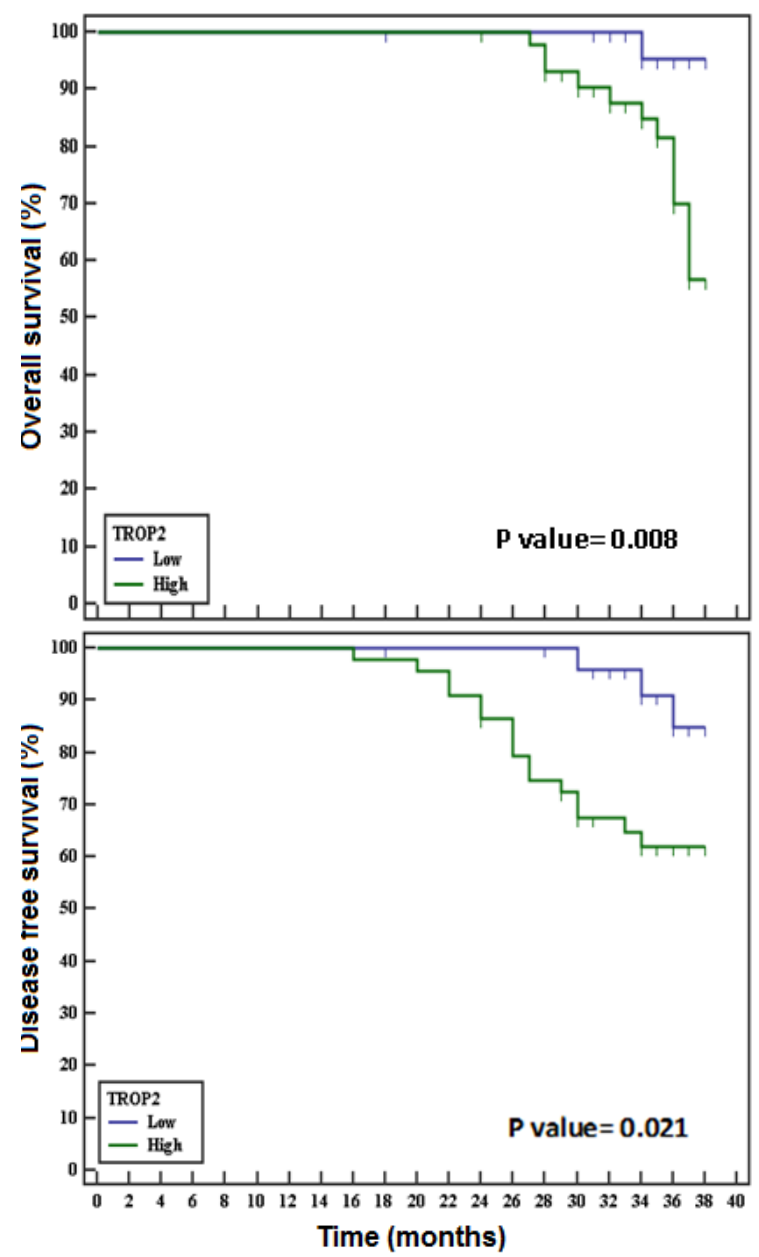

Figure 4. Kaplan-Meier survival curves for Overall survival (OS) and disease-free survival (DFS) in relation to Trop2 expression.

In the current work, Trop2 expression was significantly correlated with MVD, a finding that was consistent with Ninga, b et al. (2013) and Guo et al. (2017) who detected a positive correlation between Trop2 expression and tumor neovascularization in cholangiocarcinoma, glioma and non-small cell lung cancer (NSCLC), respectively. The exact mechanisms and factors enhancing pathological tumor angiogenesis are still unclear. However, it is suggested that binding of vascular endothelial growth factor (VEGF) to its receptors stimulates intracellular pathways including extracellular signal-regulated kinase (ERK) and phosphatidyl inositol3 kinase (PI3K), which in turn enhance angiogenic switch in several malignancies including breast cancer (Taneja et al., 2010).

Guo et al. (2017) reported that Trop2 can stimulate angiogenesis in NSCLC via activation of extracellular signal-regulated kinase /mitogen-activated protein kinase (ERK/MPAK) pathway. Moreover, they demonstrated a reduction of angiogenic markers (CD31, CD34) expression and Ki67 proliferation index after silencing Trop2 gene in vivo. Furthermore, Trop2 is associated with stem cell characters and cellular self-renewal, a process that may be linked to angiogenesis (Shvartsur and Bonavida, 2015). The structural similarity between Trop2 and EpCAM can be another explanation for its association with angiogenesis. EpCAM expression has been linked to increase MVD in hepatocellular carcinoma, which may be related to activation of WNT/B Catenin signaling pathway (Shan et al., 2011).

Trop2 is an attractive therapeutic target in several malignancies. However, targeting Trop2 requires selective strategies as it is expressed in a variety of normal tissues as well (Stepan et al., 2011). Antibody-drug conjugate (ADC) is a recent method which selectively delivers therapeutic agents attached to an antibody into target cells carrying certain antigen. Sacituzumab govitecan (IMMU-132), a recent anti Trop2 antibody linked to topoisomeraseInhibitor, irinotecan metabolite, SN-38, has gained fast track FDA approval in April 2020 owing to its promising results in clinical trials against metastatic TNBC and NSCLC (Cocco et al., 2020). It is expected that the expression level of Trop2 can affect response to ADC therapy. However, tumor response to target ADC can also be affected by other factors including tumor sensitivity to the conjugated drug (Goldenberg et al., 2018).

\section{CONCLUSION}

Trop2 expression in breast cancer was related to lower survival rates and poor prognostic parameters, in terms of high tumor grade, advanced tumor stage, lymph node invasion, lymphovascular invasion and high proliferation index. Moreover, high Trop2 expression was significantly higher among TNBC, for which it is considered as a promising therapeutic target. Additionally, Trop2 expression was significantly correlated to MVD. We speculate that the enhancement of angiogenesis is one of the mechanisms involved in Trop2 induced tumor invasion and metastasis. 
Further large-scale studies to determine molecular pathways regulating the relation between Trop2 and angiogenesis in breast cancer are required. Moreover, the effect of Trop2 targeted therapy on tumor angiogenesis should be considered in clinical trials.

\section{ABBREVIATIONS}

$A D C$ : Antibody-drug conjugate; AJCC : American Joint Committee of Cancer staging; BC: Breast cancer; $\mathrm{Cl}$ : Confidence interval; $\mathrm{DAB}$ : Diaminobenzidine; DFS: disease-free survival; ER: Estrogen receptor; EMT: Epithelialmesenchymal transition; ERK/MPAK: extracellular signal-regulated kinase /mitogenactivated protein kinase; FDA: Food and drug administration; Her2 /neu: Human epidermal growth factor receptor 2, HRP: Horseradish peroxidase; HR: hazardous ratio; IDC: invasive ductal carcinoma; LN: Lymph node; MVD: Microvascular density; NSCLC: non-small cell lung cancer; $\mathrm{N}$ : lymph node stage OS: overall survival; PI3K: phosphatidyl inositol 3 kinase; PR: Progesterone receptor; RT- PCR: reverse transcriptase polymerase chain reaction; SD: standard deviation; SPSS: Statistical Package for Social Science; T: tumor size, TACSTD2: tumourassociated calcium signal transductor, Trop2: Trophoblastic Cell Surface Protein 2; TNBC: Triple-negative breast carcinoma; VEGF: Vascular endothelial growth factor; WHO: World Health Organization.

\section{CONFLICTS OF INTEREST}

All authors have approved this article and declare no conflicts of interest.

\section{FUND}

No fund was received for this work.

\section{REFERENCES}

Abd El-Azeem MA. (2021). Glucose Transporter 4 and Interleukin 8 expression in hormone receptornegative/HER2 overexpressing breast carcinoma subtype: Correlation with the biological behavior of the tumor cells and prognostic parameters. International Journal of Cancer and Biomedical Research, 5(2):181-192.

Alberti S, Guerra E, Lattanzio R, Boujnah K, Garbo V, Moschella D, Altomare R and Trerotola M (2020). Functional inactivation of E-cadherin by Trop-2 drives cancer metastasis. Ann. Oncol, 31: S1234S1235.
Ambrogi F, Fornili $M$, Boracchi $P$, Trerotola $M$, Relli V, Simeone P, La Sorda R, Lattanzio R, Querzoli P, Pedriali $M$, Piantelli $M$, Biganzoli $E$ and Alberti $S$ (2014). Trop-2 is a determinant of breast cancer survival. PLoS One. 9 (5): e96993.

Chen Z, Xu S, Xu W, Huang J, Zhang GU, Lei L, Shao X and Wang $X$ (2015). Expression of cluster of differentiation 34 and vascular endothelial growth factor in breast cancer, and their prognostic significance. Oncol Lett.10(2):723-729.

Cocco S, Piezzo M, Calabrese A, Cianniello D, Caputo R, Lauro VD, Fusco G, Gioia GD, Licenziato $M$ and Laurentiis M (2020). Biomarkers in Triple-Negative Breast Cancer: State-of-the-Art and Future Perspectives. Int J Mol Sci.21(13):4579.

Goldenberg DM, Stein R and Sharkey RM (2018). The emergence of trophoblast cell-surface antigen 2 (TROP-2) as a novel cancer target. Oncotarget. 9 (48):28989-29006.

Goldhirsch A, Wood WC, Coates AS, Gelber RD, Thürlimann B, Senn HJ and Panel Members (2011). Strategies for subtypes--dealing with the diversity of breast cancer: highlights of the St. Gallen International Expert Consensus on the Primary Therapy of Early Breast Cancer 2011. Ann Oncol. 22 (8):1736-1747

Guo X, Zhu X, Zhao L, Li X, Cheng D and Feng K (2017). Tumor-associated calcium signal transducer 2 regulates neovascularization of non-small-cell lung cancer via activating ERK1/2 signaling pathway. Tumor Biol. 39 (3):10104283-17694324.

Huang H, Groth J, Sossey-Alaoui K, Hawthorn L, Beall S and Geradts J (2005). Aberrant expression of novel and previously described cell membrane markers in human breast cancer cell lines and tumors. Clin Cancer Res. 11(12):4357-64.

Hortobagyi G. N., Edge SB and Giuliano A (2018). New and important changes in the TNM staging system for breast cancer. ASCO Educational Book. 38: 457467.

Kluger HM, Kluger Y, Gilmore-Hebert M, DiVito K, Chang JT, Rodov S, Mironenko O, Kacinski BM, Perkins AS and Sapi E (2004). cDNA microarray analysis of invasive and tumorigenic phenotypes in a breast cancer model. Lab Invest. 84 (3):320-31.

Kraby MR, Krüger K, Opdahl S, Vatten LJ, Akslen LA and Bofin AM (2015). Microvascular proliferation in luminal $A$ and basal-like breast cancer subtypes. J Clin Pathol. 68 (11):891-7.

Kraby MR, Opdahl S, Akslen LA and Bofin AM (2017). Quantifying tumor vascularity in non-luminal breast cancers. J Clin Pathol. 70 (9):766-774.

Lakhani SE, Ellis IO, Schnitt SJ, Tan PH and van de Vijver MJ (2012). WHO classification of tumors of the breast, vol. 4. Lyon, France: International Agency for Research on Cancer (IARC).

Lin H, Huang JF, Qiu JR, Zhang HL, Tang XJ, Li H, Wang CJ, Wang ZC, Feng ZQ and Zhu J (2013). Significantly upregulated TACSTD2 and Cyclin D1 correlate with 
poor prognosis of invasive ductal breast cancer. Exp Mol Pathol. 94 (1):73-8.

Mackey JR, Kerbel RS, Gelmon KA, McLeod DM, Chia SK, Rayson D, Verma S, Collins LL, Paterson AH, Robidoux A and Pritchard KI (2012). Controlling angiogenesis in breast cancer: a systematic review of anti-angiogenic trials. Cancer Treat Rev. 38(6):673-88

Ning S, Guo S, Xie J, Xu Y, Lu X and Chen Y (2013). TROP2 correlates with microvessel density and poor prognosis in hilar cholangiocarcinoma. J Gastrointest Surg. 17(2):360-8.

Ning $_{b}$ S, Liang N, Liu B, Chen X, Pang Q and Xin T (2013). TROP2 expression and its correlation with tumor proliferation and angiogenesis in human gliomas. Neurol Sci. 34(10):1745-50.

Oualla K, El-Zawahry HM, Arun B, Reuben JM, Woodward WA, Gamal El-Din H, Lim B, Mellas N, Ueno NT and Fouad TM (2017). Novel therapeutic strategies in the treatment of triple-negative breast cancer. Ther Adv Med Oncol. 9 (7):493-511.

Pradhan A, Paudyal P, Sinha A and Agrawal CS (2017). Grading, Staging and Nottingham prognostic index of breast carcinoma. J Pathol Nepal. 7(1):1078.

Shan YF, Huang YL, Xie YK, Tan YH, Chen BC, Zhou MT, Shi $H Q$, Yu ZP, Song QT and Zhang QY (2011). Angiogenesis and clinicopathologic characteristics in different hepatocellular carcinoma subtypes defined by EpCAM and $\alpha$-fetoprotein expression status. Med Oncol. 28(4):1012-6

Shvartsur A and Bonavida B (2015). Trop2 and its overexpression in cancers: regulation and clinical/therapeutic implications. Genes Cancer. 6 (3-4): 84-105.

Stepan LP, Trueblood ES, Hale K, Babcook J, Borges L and Sutherland CL (2011). Expression of Trop2 cell surface glycoprotein in normal and tumor tissues: potential implications as a cancer therapeutic target. J Histochem Cytochem. 59 (7):701-10.

Sung H, Ferlay J, Siegel RL, Laversanne M, Soerjomataram I, Jemal A and Bray F (2021). Global Cancer Statistics 2020: Globocan Estimates of Incidence and Mortality Worldwide for 36 Cancers in 185 Countries. CA Cancer J CLIN. 71:209-249.

Taneja P, Maglic D, Kai F, Zhu S, Kendig RD, Fry EA and Inoue K (2010). Classical and Novel Prognostic Markers for Breast Cancer and their Clinical Significance. Clin Med Insights Oncol. 4:15-34

Zhao W, Jia L, Kuai X, Tang Q, Huang X, Yang T, Qiu Z, Zhu J, Huang J, Huang $W$ and Feng $Z$ (2019). The role and molecular mechanism of Trop2 induced epithelial-mesenchymal transition through mediated $\beta$-catenin in gastric cancer. Cancer Med. 8 (3):1135-1147.

Zhao W, Kuai X, Zhou X, Jia L, Wang J, Yang X, Tian Z, Wang X, Lv Q, Wang B, Zhao Y and Huang W (2018). Trop2 is a potential biomarker for the promotion of EMT in human breast cancer. Oncol Rep. 40(2):759766.

Zhou D, Cheng SQ, Ji HF, Wang JS, Xu HT, Zhang GQ and Pang D (2010). Evaluation of protein pigment epithelium-derived factor (PEDF) and microvessel density (MVD) as prognostic indicators in breast cancer. J Cancer Res Clin Oncol. 136 (11):1719-27. 